\title{
Pertinencia social de universidades públicas y privadas: perspectiva de los docentes
}

\author{
José Ángel Vera-Noriega * \\ Claudia Karina Rodríguez-Carvajal ${ }^{\star *}$ \\ Ivett Alejandra Bustamante-Castro ${ }^{* * *}$
}

* Doctor en Psicología Social. Investigador titular, Centro de Investigación en Alimentación y Desarrollo A. C. Hermosillo, México.

Correo electrónico: avera@ciad.mx

** Maestra en Desarrollo Regional. Directora de tecnoestata, $\mathrm{s}$. C.

Correo: tecnoestata@gmail.com

*** Licenciada en Psicología. Apoyo técnico,

Centro de Investigación en Alimentación y Desarrollo A. C. Hermosillo, México. Correo electrónico: ivettbustamante@ gmail.com

Recibido: 12 de abril del 2016

Aprobado: 20 de junio del 2016

Cómo citar este artículo: Vera-Noriega, José Ángel, Claudia Rodríguez-Carvajal e Ivett Bustamante-Castro. "Pertinencia social de universidades públicas y privadas: perspectiva de los docentes". Rastros Rostros 18.33

(2016): xx-xx. Impreso. doi: http://dx.doi. org/10.16925/ra.v18i33.1668

\section{Resumen}

Introducción: una de las exigencias actuales de la educación superior, tanto pública como privada, es la calidad. Dicho concepto se vincula estrechamente con la pertinencia social, la cual se refiere al papel que la educación superior desempeña en la sociedad y lo que esta espera de aquélla. En este sentido, la calidad de un sistema universitario es el producto de varios factores y procesos, entre ellos se encuentra el docente, quien es sujeto de estudio del presente trabajo, en el que se analiza su percepción sobre la pertinencia social de la institución. Metodología: desde una investigación descriptiva, se analiza la percepción de 121 docentes de dos Instituciones de educación superior, una pública y otra privada en el noroeste de México. Se llevó a cabo mediante la aplicación de encuestas con respecto a la calidad de las funciones de la institución en la que trabajan, con categorías propuestas por De la Orden, Muñoz, López, Barberá y Ndabishibije, bajo la premisa de que la calidad institucional debe evaluar la coherencia entre las necesidades que requiere la sociedad y las de la institución. Resultados: en las dos instituciones los docentes evalúan de manera positiva la pertinencia de la institución. De igual manera, se observa variabilidad de resultados entre las dos instituciones. Conclusión: la investigación permite observar que la falta de difusión de las diferentes funciones que cumple la Universidad influye en la manera en que los docentes perciben dichas funciones.

Palabras clave: calidad institucional, educación superior, evaluación de docentes, funcionalidad de la universidad. 


\title{
Social relevance of public and private universities: teachers' perspective
}

\begin{abstract}
Introduction: One of the current demands of Higher Education, both public and private, is quality. This concept is closely linked with social relevance, which refers to the role that Higher Education plays in society and what society expects from it. In this sense, a university system's quality is the product of several factors and processes, amongst which the teacher appears as a subject of study, so as to analyze his perception about the social relevance of the institution. Methodology: From a descriptive research approach, we analyze the perception of 121 teachers from two Higher Education institutions in northwest Mexico, one public and one private. Surveys regarding the quality of the functions of the institution in which they work were carried out. These had categories proposed by De la Orden, Muñoz, López, Barberá and Ndabishibije, under the premise that Institutional quality should assess the coherence between the needs of society and those of the institution. Results: In both institutions teachers evaluate positively the relevance of the institution. Similarly, variability of results is observed between the two institutions. Conclusion: The research shows that the lack of diffusion or information about the different functions that the University fulfills influences the way in which teachers perceive these functions.
\end{abstract}

Keywords: institutional quality, higher education, teacher evaluation, university functionality.

\section{Pertinência social de universidades públicas e privadas: perspectiva dos docentes}

\section{Resumo}

Introdução: uma das exigências atuais da educação superior, tanto pública quanto privada, é a qualidade. Tal conceito se vincula diretamente com a pertinência social, a qual se refere ao papel que a educação superior desempenha na sociedade e o que esta espera daquela. Nesse sentido, a qualidade de um sistema universitário é o produto de vários fatores e processos, e entre eles encontra-se o docente, como sujeito de estudo para analisar como objetivo sua percepção sobre a pertinência social da instituição. Metodologia: a partir de uma investigação descritiva, analisa-se a percepção de 121 docentes de duas instituições de educação superior, uma pública e outra privada no noroeste do México. Realizou-se mediante a aplicação de pesquisas com respeito à qualidade das funções da instituição na qual trabalham, com categorias propostas por De la Orden, Muñoz, López, Barberá e Ndabishibije, sob a premissa de que a qualidade institucional deve avaliar a coerência entre as necessidades que requerem a sociedade e as da instituição. Resultados: nas duas instituições os docentes avaliam de maneira positiva a pertinência da instituição. Da mesma maneira, observa-se variabilidade de resultados entre as duas instituições. Conclusão: a investigação permite observar que a falta de difusão das diferentes funções que a Universidade cumpre influencia na maneira em que os docentes percebem tais funções.

Palavras-chave: qualidade institucional, educação superior, avaliação de docentes, funcionalidade da Universidade. 


\section{Introducción}

La educación de calidad, en todos los niveles, además de ser una necesidad imperiosa, es una demanda social ineludible. Son múltiples los factores involucrados que en combinación posibilitan llegar a este fin y que en las instituciones es condición para la formación de profesionistas y emprendedores capaces de hacer frente a la diversidad de requerimientos sociales y productivos del país.

La Organización para la Cooperación y el Desarrollo Económicos (OCDE) (Situación educativa 28) plantea que uno de los principales desafíos en América Latina y el Caribe tiene que ver con el hecho de que los países deben fortalecer sus Instituciones de Educación Superior (IES), en especial, las grandes universidades públicas, para generar una capacidad propia de producción científica y tecnológica; las universidades deben hacer mucho más para conectarse con las necesidades de su sociedad, y es un campo privilegiado para estimular procesos de generación y aplicación de conocimientos.

En el informe final de la Conferencia Mundial sobre Educación Superior (ocDE Declaración Mundial 19) se hace énfasis en la necesidad de tomar conciencia sobre la importancia fundamental de la educación superior para el desarrollo sociocultural y económico y se muestran los principales desafíos y dificultades que enfrenta este nivel educativo: la formación basada en las competencias, la mejora y conservación de la calidad de la enseñanza, la investigación y los servicios, la pertinencia de los planes de estudios, entre otros. Planteando que ningún país podrá garantizar un auténtico desarrollo interior y sostenible, si carece de IEs que cumplan con criterios de calidad que formen profesionistas completos que también cuenten con una formación humana y enfocada a las necesidades sociales de cada localidad. México contempla en el Programa Sectorial de Educación seis objetivos para articular el esfuerzo educativo durante la presente administración, cada uno acompañado de sus respectivas estrategias y líneas de acción (Secretaría de Educación Pública 41).

Uno de los objetivos del Plan Nacional de Desarrollo es fortalecer la calidad y pertinencia de la educación media superior, superior y formación para el trabajo, a fin de que contribuya al desarrollo de México, siendo una de las estrategias para alcanzarlo, continuar el desarrollo de los mecanismos para el aseguramiento de la calidad de los programas e instituciones de educación superior (Gobierno de México 67). Para ello, se requiere que los planes y programas de estudio sean apropiados, por lo que resulta prioritario conciliar la oferta educativa con las necesidades sociales y los requerimientos del sector productivo. En este sentido, se hace necesaria información sobre la calidad de la educación superior desde una perspectiva holística que integre a los empleadores, docentes, estudiantes, egresados, y además, que asuma la funcionalidad y no solo los productos como el eje de los criterios de valoración.

La OCDE (Declaración Mundial 24) refiere que la calidad debe ser en función de la adecuación entre lo que la sociedad espera de las instituciones y lo que estas hacen, requiriendo normas éticas, imparcialidad política, capacidad crítica y, al mismo tiempo, una mejor articulación con los problemas de la sociedad y del mundo del trabajo, fundando las orientaciones a largo plazo en objetivos y necesidades sociales, tomando en cuenta las culturas y la protección del medio ambiente. En este mismo sentido, Vera-Noriega et al. (Revista de Estudos 43) plantean que la calidad en la educación superior consiste en una construcción social que varía según las necesidades e intereses que presenta cada institución de educación superior, estos requerimientos pueden ser tanto internos como externos a las distintas universidades, dichos intereses reflejan las características de la sociedad que se desea en el presente y que se desea perfilar para el futuro.

El concepto de calidad, además de considerar las características históricas culturales propias de las IES y su entorno, reconoce la presencia de referentes internacionales a partir de una valoración crítica de los mismos, pero no todos los criterios internacionales y transnacionales de calidad, ni tampoco todas las estrategias de los sistemas educativos de los países ricos, serán necesariamente adecuados o importantes para las naciones pobres y en desarrollo como es el caso de los países latinoamericanos (Vera-Noriega et al. Revista de Estudos 34).

De la Orden (Revista de Pedagogía 152) define la calidad de la educación universitaria, como un conjunto de relaciones de coherencia entre los componentes de la universidad concebida como un sistema. Los componentes se refieren a: eficacia o efectividad, eficiencia y funcionalidad o pertinencia, siendo estos tres criterios centrales con los que las evaluaciones deben estar conformadas. Estos criterios dictaminados por la OCDE fueron retomados para el desarrollo de un marco referencial por medio del cual se pueden 
llevar a cabo las evaluaciones de la calidad en todos los casos y tipos de IEs, lo cual significa que la evaluación considera la diversidad que hay en cada una de las IEs y sobre todo que todas las evaluaciones tengan una misma finalidad, considerando el logro de la calidad educativa, independientemente del tipo de institución. En este sentido, es posible determinar criterios cuantificables de eficacia, eficiencia y funcionalidad para la identificación de relaciones de coherencia e incoherencia entre los elementos del sistema, por lo que resulta necesario precisar objetivamente cada una de estas dimensiones. La eficacia se define en función de la relación que existe entre los resultados educativos obtenidos y los objetivos prescritos como consecuencias del proceso educativo del sistema. La eficiencia se define a través de la correspondencia de capital y resultados, esencialmente en la valoración de los costos que suponen los objetivos del sistema y la eficiente operación del capital para conseguirlos (De la Orden Estudios sobre 29).

La funcionalidad, por otro lado, se define como "la capacidad que la institución demuestra de ajustar sus objetivos y, por tanto, toda la acción y los medios de que dispone, al cumplimiento de las funciones que le han sido asignadas por la sociedad" (De la Orden et al. Revista electrónica 8). La pertinencia de las funciones del sistema está determinada por las expectativas y necesidades del entorno al cual corresponde, es decir, si el sistema mantiene un alto grado de eficiencia y eficacia, sin satisfacer las necesidades e intereses educativos del contexto, las funciones ejecutadas carecerían de pertinencia y correspondencia con las pretensiones por las cuales fue establecido (De la Orden REVALUE 13).

En la presente investigación se tomará el criterio de funcionalidad definida por De la Orden et al. (Archivos analíticos 271) para medir la calidad universitaria, como un determinante que se origina desde fuera de las instituciones, por el contexto, los valores, las necesidades y aspiraciones tanto culturales, científicas y económicas de la entidad. Siendo entonces los insumos, los procesos y las metas en relación con las expectativas y necesidades sociales las que definen la calidad institucional de las IEs desde su funcionalidad o pertinencia.

El objetivo del presente estudio es analizar la percepción de los docentes de dos instituciones de educación superior en el noroeste de México: una pública y otra privada, siendo estas una laica y la otra católica, con respecto a categorías derivadas de las propuestas por De la Orden et al. (Revista de Educación
7), con el fin de determinar la coherencia entre las variables asociadas a la estructura y funcionamiento de las instituciones.

\section{Metodología}

\section{Características de la población}

Los participantes son docentes de diferentes áreas de formación, de la Universidad de Sonora en las áreas de ingenierías y en la Universidad Kino en el área de ingenierías y en el área de sociales. La muestra se conforma de 121 docentes: $47 \%$ son del sexo femenino y $53 \%$ son varones. Los tipos de contratos pueden ser de tres modalidades: tiempo completo, medio tiempo y horas sueltas. Sus edades se encuentran en un rango de 26 a 56 años; el $67 \%$ son de nivel licenciatura, $28 \%$ de maestría y $5 \%$ de doctorado. La mayoría de los participantes tienen de los 0 a los 5 años de antigüedad en la institución, representando el $68 \%$ de los evaluados.

\section{Descripción de instrumento}

El instrumento se compone de dos secciones. La primera consiste en datos de identificación, en la que se intenta recoger una descripción general sobre cada uno de los encuestados, mediante la obtención de algunos datos: edad, sexo, lugar de nacimiento, grado de estudios, años como docente, carrera, número de cursos de formación pedagógica, porcentaje de tiempo dedicado a la docencia, instituciones en las que ha trabajado como profesional académico y tipo de contrato.

La segunda sección tiene el objetivo de recabar la perspectiva de los docentes, en relación con la calidad de las funciones de la institución en la que trabajan. Para ello, se recurrió al instrumento de De la Orden et al. (Archivos analíticos 35), en el que se abordan diferentes aspectos del sistema universitario. El instrumento consiste en un cuestionario en el cual los docentes emiten su percepción a través de una escala tipo Likert en la que el mínimo es representado con el número 1 y el máximo con el número 7. Para efectos de esta investigación, las respuestas de los docentes, encontradas en el rango de 1 a 4 , se consideran como desacuerdo, mientras que en el caso de las respuestas encontradas en el rango de 4 a 7 son consideradas de acuerdo. 
El modelo original de funcionalidad institucional de De la Orden et al. (Archivos analíticos 11) se compone de 74 reactivos distribuidos en 29 multireactivos los cuales conforman seis dimensiones: 1) funciones de fomento y desarrollo de la ciencia; 2) funciones de fomento y desarrollo de la tecnología; 3 ) funciones de fomento y desarrollo de la actividad económica; 4) funciones de fomento, desarrollo y trasmisión de la cultura; 5) funciones de preparación para el mundo profesional o profesionalización y 6) funciones de compromiso social.

En el presente estudio, al igual que en el modelo original de De la Orden, se analizaron 29 multireactivos que conforman las seis dimensiones y los nueve indicadores haciendo un total de 74 reactivos. La primera dimensión describe el resultado de investigación en la institución, en la cual se indica la medida en que dichas investigaciones generan avances en diferentes rubros. Esta dimensión es la sumatoria de tres índices que indican los avances teóricos (R12.1), avances tecnológicos (R12.2) y patentes (R12.3).

La segunda dimensión enuncia programas y ética, y en ella se indica la medida en que la Universidad desarrolla programas o actividades paralelas a la formación de los estudiantes referidos a diferentes aspectos. Esta dimensión es la sumatoria de cinco índices, que indican aspectos referidos a la valoración social de la profesión (R5.1), el perfil profesional que se espera del alumno al terminar los estudios (R5.2), la ética profesional que se espera (R5.3), los contratos laborales (R5.4), la estructura sindical o gremial (R5.5) y la democracia (R5.6).

La tercera dimensión hace referencia a las políticas y normas e indica la medida en que refuerza algunos aspectos. Esta dimensión, es la sumatoria de 6 índices que refieren a aspectos como la formación científica (R1.1), formación tecnológica (R1.2), actividades económicas (R1.3), transmisión de la cultura (R1.4), profesionalización de los estudiantes (R1.5) y compromiso social (R1.6).

La cuarta dimensión tiene que ver con las facetas de la acción universitaria, es decir, en qué medida las políticas y normas de la universidad contribuyen al desarrollo de dichas facetas, esta dimensión, es la sumatoria de 4 índices que refieren a las facetas de investigación (R2.1), enseñanza (R2.2), extensión (R2.3) e intercambio (R2.4).

En relación con la quinta dimensión, esta refiere al programa de estudios, es decir, en qué medida responde a algunos aspectos del contexto de la Universidad. Esta dimensión es la sumatoria de tres índices, los cuales refieren aspectos como las metas de la institución (R4.1), demandas sociales (R4.2) y necesidades del entorno (R4.3).

Finalmente, la sexta dimensión hace alusión al grado de conocimiento de los alumnos de las publicaciones de la universidad, esta dimensión es la sumatoria de 7 índices, los cuales refieren a diversas publicaciones tales como libros (R13.1), revistas (R13.2), ponencias en eventos científicos (R13.3), folletos (R13.4), trípticos (R13.5), CD-ROM (R13.6) y páginas WEB (R13.7).

\section{Resultados}

Los datos recabados muestran que la mayoría de los docentes encuestados tiene de 0 a 3 cursos de formación pedagógica, siendo el $82,6 \%$ de ellos, seguido por $11,6 \%$ de 3 a 6 horas de curso, $3,3 \%$ de 6 a 10 horas y 2,5 más de 10 horas de curso. El 28,9\% de los encuestados contestó que se dedica todo su tiempo a la docencia siendo la mayoría de ellos, el 18,2\% dedica la mitad del tiempo y el $14,9 \%$ dedica el $80 \%$.

En cuanto a la gestión, el $48,8 \%$ contestó que no dedica su tiempo a dicha actividad, el 11,6\% dedica la mitad del tiempo y el 7,4\% dedica el $20 \%$. De este modo, el 50,4\% de los docentes no dedica tiempo a la investigación, el $15,7 \%$ dedica $20 \%$ y el 9,9\% de los profesores dedica el 10\% del tiempo a dicha actividad. Estos resultados indican que la actividad que realizan la mayoría de los encuestados es la docencia. El 94,2\% de los docentes ha trabajo de entre 0 a 3 instituciones y el $14 \%$ tiene 4 y 10 horas de trabajo, seguido por $6,6 \%$ de 8 horas, 5,8\% de 25 horas y 4,1\% de 2 horas.

Las características psicométricas del instrumento derivadas del análisis factorial por factores principales con rotación varimax (tabla 1), indican que para la dimensión "Resultado de investigación", se obtuvo un alfa de Cronbach de 0,78 , lo que señala la consistencia interna de la dimensión. Esta dimensión con 3 reactivos explica el 72,22 de la varianza, saturando en el primer factor el 30\% de la varianza total. Lo anterior demuestra que, de acuerdo con la percepción de los docentes de dos universidades del Noroeste de México, la funcionalidad de la institución es vista con base en los avances que genera la investigación que se realiza en la universidad.

La siguiente dimensión "Programas-Ética", constituida por seis reactivos, le corresponde una alfa de 0,88 . De este modo las siguientes cuatro dimensiones de funcionalidad: "Políticas y normas", "Acción universitaria", "Programas de estudios" y 
"Conocimiento de publicación" cuentan con alfas suficientemente altas para comprobar coherencia e intercorrelación entre reactivos.

Esta solución factorial fue decidida con base en la revisión del gráfico de sedimentación, y la matriz de componentes rotados, incluyendo los valores de peso factorial mayor y menor en cada una de las dimensiones, según el programa estadístico spss 21 . Cada uno de los reactivos presentó una cifra que indica el peso factorial y fue ubicada dentro de cada una de las seis dimensiones o componentes.
En la tabla 1 se observa, además, que la media en cada una de las dimensiones señala que existe un acuerdo mínimo con valores entre cuatro y cinco, y solo cuando la media obtiene valores entre seis y siete supondríamos altos niveles de acuerdo. La media de 3,3 debe ser interpretada como un desacuerdo mínimo promedio en los 3 reactivos que componen la dimensión. Dichos valores de las medias, fueron promediados entre los resultados de las dos universidades.

Tabla 1. Dimensiones de funcionalidad, peso factorial, valores de media, desviación estándar y alfa de Cronbach

\begin{tabular}{|l|c|c|c|c|c|c|c|c|c|}
\hline \multicolumn{1}{|c|}{ Factor } & $\begin{array}{c}\text { Ítems } \\
\text { in. }\end{array}$ & $\begin{array}{c}\text { Ítems } \\
\text { fin. }\end{array}$ & KMO & $\begin{array}{c}\% \\
\text { V. E. }\end{array}$ & $\begin{array}{c}\text { Mayor } \\
\text { PF. }\end{array}$ & $\begin{array}{c}\text { Menor } \\
\text { P. F. }\end{array}$ & $\begin{array}{c}\text { Alfa } \\
\text { Cronbach }\end{array}$ & X & D. E. \\
\hline 1.Resultado de investigación & 3 & 3 & 0,59 & 72,22 & 0,92 & 0,78 & 0,78 & 3,35 & 1,14 \\
\hline 2. Programas-Ética & 6 & 6 & 0,75 & 65,55 & 0,94 & 0,69 & 0,88 & 4,80 & 1,18 \\
\hline 3.Políticas y normas & 6 & 4 & 0,64 & 58,48 & 0,89 & 0,64 & 0,73 & 4,92 & 1,12 \\
\hline 4.Acción universitaria & 4 & 4 & 0,71 & 62,96 & 0,91 & 0,64 & 0,78 & 4,87 & 1,35 \\
\hline 5.Programa de estudios & 3 & 3 & 0,61 & 67,63 & 0,89 & 0,74 & 0,76 & 4,10 & 0,77 \\
\hline 6. Conocimiento publicaciones & 7 & 5 & 0,82 & 59,45 & 0,85 & 0,67 & 0,80 & 4,70 & 1,32 \\
\hline
\end{tabular}

Ítems in. Es el número de reactivos iniciales, Ítems fin. Es el número de reactivos finales, V.E. es varianza explicada, X es la media, D.E. es la desviación estándar y P.F. significa peso factorial.

Fuente: elaboración propia con base en los resultados de la aplicación de encuestas de este estudio.

En la dimensión de funcionalidad la escala de 7 puntos reporta un punto medio siendo este el 4 y por debajo de la percepción de cada una de las dimensiones indica un proceso de acuerdo con los esfuerzos de acuerdo con la institución y por arriba de 4 el conocimiento de la medida para lograrlo. De esta forma, los valores de 4 a 5 implican una percepción baja (pb) aunque afirmativa de la medida con la cual la universidad se ocupa de la dimensión, de 5 a 6 es moderada (pm) y de 6 a 7 es alta (pa).

En todas las dimensiones, los docentes de la Universidad Kino (UnIKINo) y de la Universidad de Sonora (UNISON) coinciden en aprobar las funciones de la Institución en todas las dimensiones, pues sus promedios se encuentran por arriba de 4 , es decir, los docentes perciben en todas ellas que la universidad refuerza aspectos de la formación de los alumnos, política, planes de estudio, acción universitaria y conocimiento de publicaciones pero sin diferencias significativas entre las dos instituciones (tabla 2).

Continuando con el análisis, la dimensión "Resultado de investigaciones" fue calificada como baja por parte de los docentes de las dos instituciones, presentando menor puntuación la UNISON con una media de 3,41 y unikino 3,91, lo cual indica que los profesores consideran que la institución está poco motivada para promover avances teóricos, tecnológicos y patentes. Finalmente, la dimensión referida a la medida en la cual la universidad genera avances teóricos, tecnológicos y patentes a partir de la investigación presenta diferencia significativa $(\mathrm{t}=-2,625 ; \mathrm{gl}=$ 119; $\mathrm{p}=$,010). Se realizó un bootstrap para 1000 sujetos, obteniendo un nivel de significancia de 0,004.

Tabla 2. Promedios de cada una de las dimensiones de funcionalidad institucional para las dos instituciones de educación superior

\begin{tabular}{|l|c|c|c|c|c|}
\hline $\begin{array}{c}\text { Dimensiones de funcionali- } \\
\text { dad institucional }\end{array}$ & \multicolumn{2}{|c|}{ unison } & \multicolumn{2}{c|}{ unikino } & Sig. \\
\hline & Media & $\mathrm{R}$ & Media & $\mathrm{R}$ & \\
\hline 1.Resultado de investigación & 3,41 & $\mathrm{~Pb}$ & 3,91 & $\mathrm{~Pb}$ &, 010 \\
\hline 2. Programas-Ética & 4,25 & $\mathrm{~Pb}$ & 4,00 & $\mathrm{~Pb}$ &, 102 \\
\hline 3.Políticas y normas & 4,87 & $\mathrm{~Pb}$ & 5,04 & $\mathrm{Pm}$ &, 332 \\
\hline 4.Acción universitaria & 3,94 & $\mathrm{~Pb}$ & 3,86 & $\mathrm{~Pb}$ &, 670 \\
\hline 5.Programa de estudios & 4,24 & $\mathrm{~Pb}$ & 4,31 & $\mathrm{~Pb}$ &, 535 \\
\hline $\begin{array}{l}\text { 6.Conocimiento publica- } \\
\text { ciones }\end{array}$ & 3,85 & $\mathrm{~Pb}$ & 3,92 & $\mathrm{~Pb}$ &, 710 \\
\hline
\end{tabular}

$\mathrm{R}=$ Resultado, $\mathrm{pb}=$ percepción baja, $\mathrm{pm}=$ percepción moderada. Fuente: elaboración propia. 
La tabla 3 representa los resultados de las dos instituciones de educación superior para cada uno de los indicadores del modelo, De la Orden et.al. (Archivos Analíticos 35), en la cual el reactivo, "recursos con los que cuenta la universidad", presenta una percepción moderada para las dos universidades con una media de 5,26 para UNISON y UNIKINO, lo cual sugiere que los docentes perciben que la universidad cumple mejor con el compromiso de tener los recursos para resolver problemas. De este modo, el indicador "programas de intercambio con otras universidades" presenta una percepción moderada en los docentes de la universidad pública con una media de 5,17 y una percepción baja para la universidad privada. Este indicador presenta una diferencia significativa $(\mathrm{t}=2,744 ; \mathrm{gl}=$ 119; $\mathrm{p}=, 007)$ entre la percepción de los docentes en relación con la existencia de programas de intercambio con otras universidades.

El indicador que calificó con la media de percepción más baja fue "participación en actividades que encaminan a la formación social" con una media de 3,78 en el caso de unison y 3,79 para UnIKINO, dichos resultados indican que los docentes participan con poca frecuencia en actividades de la universidad que se encaminan al desarrollo o perfeccionamiento de su formación social. Todos los demás se encuentran en grado de aceptación sin diferencias significativas.

Se llevó a cabo un análisis de varianza de una sola vía de efectos aleatorios, para comparar las diferentes dimensiones de la funcionalidad institucional por sexo, edad, grado de estudios, años como docente y años en la institución para cada uno de los profesores de ambas universidades. Se compararon cuatro rangos de edad, a saber: el primer rango de 26 a 36 años de edad, el segundo rango de 37 a 38 años de edad, el tercer rango de 39 a 43 años de edad y en cuarto rango de 45 a 56 años de edad. En la comparación paramétrica politómica se encontró que la dimensión "resultados de investigación" es la única que resulto significativa, particularmente el caso de la percepción de cumplimiento que perciben los docentes más jóvenes con una media de 5,23.

Cuando se probó el efecto del grado de estudios, comparando únicamente licenciatura con posgrado, se encontró una diferencia significativa en relación con la aceptación de la funcionalidad del programa de estudios particularmente para aquellos que tienen posgrado. El factor sexo no tuvo ningún impacto sobre las dimensiones de funcionalidad.
Tabla 3. Promedios de cada uno de los indicadores de funcionalidad institucional para las dos instituciones de educación superior

\begin{tabular}{|l|c|c|c|c|c|}
\hline $\begin{array}{l}\text { Indicadores de funciona- } \\
\text { lidad institucional }\end{array}$ & \multicolumn{2}{|c|}{ UNIsON } & \multicolumn{2}{|c|}{ UNIKINO } & Sig. \\
\hline & Media & $\mathrm{R}$ & Media & $\mathrm{R}$ & \\
\hline $\begin{array}{l}\text { 1. Recursos con los que } \\
\text { cuenta la universidad. }\end{array}$ & 5,26 & $\mathrm{Pm}$ & 5,26 & $\mathrm{Pm}$ &, 999 \\
\hline $\begin{array}{l}\text { 2. Con qué frecuencia } \\
\text { se desarrollan progra- } \\
\text { mas para dar solución a } \\
\text { problemas. }\end{array}$ & 4,60 & $\mathrm{~Pb}$ & 4,44 & $\mathrm{~Pb}$ &, 497 \\
\hline $\begin{array}{l}\text { 3. Programas de intercam- } \\
\text { bio con otras universi- } \\
\text { dades. }\end{array}$ & 5,30 & $\mathrm{Pm}$ & 4,53 & $\mathrm{~Pb}$ &, 007 \\
\hline $\begin{array}{l}\text { 4. Participación en activi- } \\
\text { dades que encaminan a la } \\
\text { formación social. }\end{array}$ & 3,78 & $\mathrm{~Pb}$ & 3,76 & $\mathrm{~Pb}$ &, 950 \\
\hline $\begin{array}{l}\text { 5. Nivel de la universidad } \\
\text { en trabajos comunitarios. }\end{array}$ & 4,31 & $\mathrm{~Pb}$ & 4,47 & $\mathrm{~Pb}$ &, 574 \\
\hline $\begin{array}{l}\text { 6. Titulados manifies- } \\
\text { tan compromiso con } \\
\text { principios y valores de sus } \\
\text { profesiones. }\end{array}$ & 4,15 & $\mathrm{~Pb}$ & 4,59 & $\mathrm{~Pb}$ &, 130 \\
\hline $\begin{array}{l}\text { 7. Con qué frecuencia } \\
\text { participas en proyectos de } \\
\text { investigación. }\end{array}$ & 4,36 & $\mathrm{~Pb}$ & 4,47 & $\mathrm{~Pb}$ &, 664 \\
\hline $\begin{array}{l}\text { 8. Grado de satisfacción } \\
\text { de la formación recibida } \\
\text { en la universidad. }\end{array}$ & 5,17 & $\mathrm{Pm}$ & 4,91 & $\mathrm{~Pb}$ &, 391 \\
\hline $\begin{array}{l}\text { 9. Ofertas de la universi- } \\
\text { dad para intercambio en } \\
\text { otras universidades. }\end{array}$ & 4,48 & $\mathrm{~Pb}$ & 4,09 & $\mathrm{~Pb}$ &, 248 \\
\hline
\end{tabular}

$\mathrm{R}=$ Resultado, $\mathrm{pb}=$ percepción baja, $\mathrm{pm}=$ percepción moderada. Fuente: elaboración propia.

Este fenómeno se repite para la dimensión "políticas y normas", en la que los más jóvenes se muestran más optimistas con el cumplimiento de esta función de la universidad, lo mismo que con la dimensión "conocimiento de las publicaciones" que genera la universidad, y siguen siendo las personas con mayor experiencia docente las que perciben con menor completitud estas funciones dentro de la universidad.

Por otro lado, se evaluó el impacto de los años como docente considerando tres rangos: el primer rango de 0 a 5 años, el segundo rango de 6 a 10 años y el tercer rango de 11 a 30 años; encontrándose que son los más jóvenes los que tienen mayor aceptación en relación con el cumplimiento de los "resultados de la investigación”, sin embargo, hay que hacer notar en esta dimensión que los docentes con mayor número 
de años presentan las tasas de incredulidad más elevadas, con valores de 2,67.

Es importante señalar que cuando se colocan como factor los años en la institución los cuales están trazados en tres rangos: el primero de 0 a 5 años, el segundo de 6 a 10 años y el tercero de 11 a 30 años; encontramos en "resultados de la investigación" una diferencia vinculada de nuevo con aquellos que tienen más años en la institución, mostrando una incredulidad de 2,6. Esta percepción de bajo cumplimiento también se repite para las funciones de "políticas y normas" y los "conocimientos de publicación", siguiendo esta lógica, los jóvenes son los que tienen una percepción de cumplimiento mientras que las personas con mayores años en la Institución perciben que estas tres funciones no se están cumpliendo cabalmente dentro de la universidad.

Continuando con los análisis de varianza de una sola vía de efectos aleatorios para la edad, el sexo, los años en la docencia, el grado académico y los años en la institución, comparamos los nueve indicadores que se encuentran dentro del modelo de funcionalidad, de la Orden et. al. (Archivos Analíticos 35), que son: el grado de satisfacción con la universidad, las ofertas de intercambio, los programas de intercambio, la formación social, el trabajo comunitario, el compromiso con valores, la participación en proyectos de investigación, los programas para solucionar problemas sociales y los recursos de la universidad. Cuando utilizamos como factor la edad, se encontró que solamente la percepción acerca de los recursos con los que cuenta la universidad resulta significativo, desarrollando una lógica en la cual las personas de mayor edad asumen que la universidad cumple mejor con el compromiso de tener los recursos para resolver los problemas, que aquellos que tienen menor edad los cuales tienen una media de 4,48.

Al comparar la variable "sexo", como factor con los nueve indicadores del modelo de funcionalidad, se encontró que para ninguno de ellos genera alguna diferencia significativa, sin embargo, al continuar con el grado académico como factor, diferenciando solamente licenciatura con posgrado, encontramos que los valores y principios de las profesiones, la participación en proyectos de investigación, los programas para solucionar problemas sociales y los recursos con los que cuenta la universidad estuvieron relacionados con niveles de significancia de ,001 en relación con el grado académico, particularmente fueron los docentes que solamente tienen licenciatura aquellos que indicaron tasas promedio de completitud comparado con los de posgrado.

En relación con los años como docente únicamente es significativo para los recursos con los que cuenta la universidad con una significancia de, 01 y que particularmente está asociado en la post hoc, a los docentes que tienen mayor cantidad de años en la docencia, dado que ellos son los que perciben que este factor tiene una menor completitud y es en menor medida atendido por la institución.

Por otro lado, al observar los años en la institución como factor y los nueve indicadores como variable, señala una lógica idéntica en relación con los años de docencia y las dimensiones de funcionalidad, particularmente el compromiso con los principios y valores de sus profesiones, la frecuencia con la que apoyan la participación en los proyectos de investigación en los proyectos para dar soluciones a programas sociales, y los recursos con los que cuenta la universidad con un resultado significativo al ,01, en todos los casos fueron las personas con mayor cantidad de años en la institución aquellos que percibieron estos indicadores con tasas menores de completitud para el funcionamiento de la universidad.

\section{Conclusiones}

Las instituciones de educación superior poseen diferentes políticas institucionales que rigen el funcionamiento de las mismas, además los procesos educativos que se llevan a cabo son particulares del tipo de iEs a la cual pertenecen. A continuación, se presentan los puntos comparativos entre las dos universidades:

a. Misión: Unison, a través de alianzas, apoya y asegura que las comunidades regionales apliquen conocimiento y tecnología que permita el desarrollo exitoso de su infraestructura cultural, social y económica, resultando en un ambiente que provee vida sustentable y oportunidades a sus habitantes (UNISON párr. 1). Universidad Kino se compromete a educar integralmente a personas que busquen mejorar su calidad de vida, formando emprendedores altamente calificados, competentes a nivel global, con una adecuada concepción de su realidad y de la responsabilidad que le corresponde asumir en el desarrollo de su región (UNIKINO párr. 4).

b. Visión: unison es parte de una sociedad que continuamente mejora la supervivencia, salud, 
autosuficiencia y bienestar de sus ciudadanos, generando contribuciones de alto valor agregado a la sociedad y economía del conocimiento (UNISON párr. 1). Mientras que la Universidad Kino, en el 2025, aspira ser una institución educativa definida en los principios del humanismo de inspiración católica más reconocida en el país, por su liderazgo en la formación de personas emprendedoras y altamente calificadas (UNIKINo párr. 5)

c. Organización: UNISON se conforma por: rectoría, direcciones académicas, direcciones administrativas, campus, departamentos/coordinaciones académicas, y departamentos/coordinaciones administrativas (UnIson párr. 1) Por otro lado, la Universidad Kino se conforma por: rectoría, direcciones académicas, campus y departamentos

d. Valores: Unison considera valores como: bien común, integridad, honradez, imparcialidad, trasparencia y rendición de cuentas, entorno cultural y ecológico, generosidad, igualdad, respeto y liderazgo (UNISON párr. 1). La Universidad Kino toma en cuenta valores como: calidad, fe, justicia, respeto, responsabilidad y sustentabilidad (UNIKINO párr. 3)

Aunque cada una de las instituciones tiene un enfoque específico, tienen un punto importante en común, buscan que su misión como universidad se desenvuelva hacia el desarrollo de una vida sustentable; es decir, que los programas sean pertinentes con las necesidades de la sociedad. Por el contrario, la visión tiene una orientación distinta, UNISON busca contribuir a la sociedad, pretende ser una IEs líder en el estado, UNIKINo se orienta hacia el humanismo, esto debido a que cada una es de diferente tipo. Por lo mismo, en lo que se refiere a los valores, cada una de las instituciones desarrolla diferentes valores dependiendo del enfoque de la universidad.

De manera general, se puede observar que los profesores de las dos universidades evalúan positivamente la pertinencia de la institución. Cabe señalar que existen diferencias entre las variables de contexto que se manejan contrastadas con las variables de funcionalidad. Un problema que puede generar confusión en los datos está relacionado con el conocimiento que el profesor tiene de las actividades de su propia universidad y por ello, la difusión de las diferentes funciones es fundamental en la respuesta del docente. Los años como docentes pueden convertirse en un factor relacionado con este conocimiento de las funciones y de manera paralela los sistemas de inducción para el personal docentes (Vera-Noriega et al. Ra Ximhai 63).

En relación con los indicadores, es interesante hacer ver que la universidad estatal con todos sus recursos, establece diferencias significativas en las posibilidades de intercambio con otras universidades, comparada con la universidad particular que no es corporativa. Por otro lado, la percepción de los docentes acerca de los recursos de la universidad es prácticamente idéntica, lo mismo, en relación con los programas para resolver problemas sociales. Se puede observar, que en las dos universidades las percepciones son muy bajas, y los docentes asumen que en escasa medida se cumple con la participación de actividades que encaminan a la formación social de los estudiantes. Esta percepción por parte de los estudiantes sobre su función social se ha subestimado y forma parte del conjunto de variables asociadas al rezago educativo (Vera-Noriega et al. Universidades 43).

Respecto a los indicadores de grado de satisfacción de la formación recibida, los principios y valores de los egresados, y la participación en proyectos de investigación, no existen diferencias significativas en las dos universidades, lo cual, nos hace pensar sobre la inversión pública en las universidades estatales o federales, las cuales, resultan con un mismo nivel de percepción deficiente por parte de sus docentes y no se encuentran diferencias en relación con las universidades privadas. "Resultados de investigación" resultó significativamente diferente en relación con la percepción por edad, siendo los más jóvenes aquellos que perciben con mejores niveles de completitud esta función.

Por otro lado, podríamos asumir que una variable que impacta la percepción en las diferentes funciones tiene que ver con la edad en la docencia, es decir, los jóvenes perciben con mayor completitud dichas funciones, que aquellos que tienen de 11 a 30 años de antigüedad.

Continuando con la comparación de variables de atributo en los docentes y los indicadores, se encontró que, a mayor cantidad de años en docencia se percibe menor completitud en relación con los recursos con los que cuenta la universidad, al igual que con los indicadores de participación en proyectos de investigación, solución de problemas sociales. Con base en dichos resultados, se puede resumir que en relación con los indicadores la edad es un factor importante, y que en la medida en que esta incrementa es más escasa la completitud de la percepción de estos indicadores en los docentes. 
El "conocimiento de las publicaciones" y la "acción universitaria" relacionada con los programas sociales, tal como lo decíamos, la formación de valores y los programas de intercambio resultan ser los elementos fundamentales que deben estimularse y desarrollarse en ambas universidades. En un estudio anterior (Vera-Noriega, Universidades 14) se concluía que el menor nivel de desarrollo percibido por los docentes es con la competencia relacionada con la divulgación de resultados de las actividades científicas y tecnológicas y la baja productividad académica en los docentes y escasas oportunidades para desarrollar investigación y su relación con actividades de ciencia e investigación y variables tales como la productividad docente y las oportunidades para investigar.

Parece necesario, en las dos universidades, mejorar todas las actividades que van encaminadas a la formación social del estudiante, y que tienen que ver con la construcción de los alumnos como ciudadanos en términos de valores y capacidad de control de emociones. Tal como se puede observar, la percepción de completitud es baja en la mayoría de las dimensiones, pero particularmente las que tienen que ver con valores y principios de los egresados, así como también, las que se relacionan con trabajos comunitarios y formación social, junto con las posibilidades y programas de intercambio con otras universidades, las cuales, son urgentes que sean atendidas por parte de las instituciones para ganar credibilidad.

Todo indica que las diferencias son muy sutiles entre las dos universidades, y no parece, desde el punto de vista de los docentes, existir gran diferencia a la hora de evaluar la percepción de la funcionalidad y de los indicadores asociados en las dos. Lo que resulta más importante son las comparaciones en atributos, considerando que los profesores con mayor antigüedad dentro de la institución perciben cada vez menor completitud de las funciones comparada con los jóvenes, y por otro lado los docentes con posgrado reconocen algunas de las funciones y de los indicadores con mayor completitud que aquellos que tienen licenciatura.

\section{Referencias}

Gobierno de México. Plan Nacional de Desarrollo 20132018. México: Editorial de Gobierno, 2013. Web. 3 de enero del 2016.
Secretaría de Educación Pública. Programa Sectorial de Educación 2013-2018. México: Secretaría de Educación Pública, 2013. Web. 3 de enero del 2016.

De la Orden, Arturo. "La calidad de la educación”. Bordón. Revista de Pedagogía 40.2 (1988): 149-162. Web. 5 de noviembre del 2015 .

De la Orden, Arturo. "Desarrollo y validación de un modelo de calidad universitaria como base para su evaluación". Revista Electrónica de Investigación y Evaluación Educativa 3.1-2 (1997). Web. 15 de noviembre del 2015.

De la Orden, Arturo, et al. "Funciones de la universidad". Revista de Educación 328 (2002): 267-281. Web. 13 de noviembre del 2015.

De la Orden, Arturo, et al. "Niveles y perfiles de funcionalidad como dimensión de calidad universitaria. Un estudio empírico en la Universidad Complutense". Archivos Analíticos de Políticas 15.12 (2007). Impreso.

De la Orden, Arturo. "Evaluación y calidad: análisis de un modelo”. Estudios sobre Educación 16 (2009). Web. 9 de diciembre del 2015.

De la Orden, Arturo. "Innovación, evaluación y calidad en la educación”. REVALUE 1.1 (2012). Web. 3 de enero del 2015.

Organización para la Cooperación y el Desarrollo Económico (OCDE). Declaración mundial sobre la educación superior en el siglo XXI: visión y acción. Y Marco de acción prioritaria para el cambio y el desarrollo de la educación superior. Francia: Ediciones OCDE, 1998. Web. 30 de enero del 2016.

Organización para la Cooperación y el Desarrollo Económico (OCDE). Situación educativa de América Latina y el Caribe: hacia la educación de calidad para todos al 2015. Chile: Ediciones del Imbunche, 2013. Web. 30 de enero del 2016.

unikino. “Acerca de Universidad Kino: Misión”. Universidad Kino, s. f. Web. 3 marzo del 2016.

unison. "Misión Institucional". Universidad de Sonora, s. f. Web. 3 marzo del 2016.

UNISON "Visión”. Universidad de Sonora, s. f. Web. 3 marzo del 2016.

unison. "Contraloría General: Organigrama. Universidad de Sonora, s. f. Web. 3 marzo del 2016.

UnIson. "Valores". Universidad de Sonora. s.f. Web. 3 marzo del 2016

Vera-Noriega, José Ángel, María del Carmen Islas Sepúlveda y Claudia Karina Rodríguez. “Tipología de la Prácti- 
ca Docente en Educación Superior Tecnológica”. $R a$ Ximhai 4.2 (2008): 49-66. Web. 15 de febrero del 2016.

Vera-Noriega, José Ángel, Etty-Haydeé Estevez-Nenninger, Luz del Carmen Ayón-Munguía y Maira-Fernanda Hurtado-Abril. "Pertinencia Social de las Instituciones de Educación Superior con Base en la Percepción de los Estudiantes Universitarios". Revista de Estudos e Pesquisas sobre as Américas 5.1 (2011): 33-44. Web. 10 de febrero del 2016.
Vera-Noriega, et al. "Factores asociados al rezago en estudiantes de una institución de educación superior en México". Revista Iberoamericana de Educación Superior 3.7 (2012): 41-56. Impreso.

Vera-Noriega, José Ángel. "Competencias científicas de docentes de educación superior tecnológica en México". Universidades 62.52 (2012): 4-17. Impreso. 